Conclusions Although MT has proven to be an effective treatment for AIS, it appears that prior to MR CLEAN, patients who underwent MT had a higher likelihood of requiring a hemicraniectomy during the same hospital admission. Since MTs were not standardized prior to 2014 , the variability in procedural technique could ultimately cause unreliable degrees of recanalization and thus lead to varied outcomes. Therefore, the effect of MT on hemicraniectomy rates in patients with AIS warrants further investigation following the advent of standardized thrombectomy techniques.

Disclosures E. Luther: None. D. McCarthy: None. R. Starke: None.

\section{E-100 PREDICTORS OF UNFAVORABLE OUTCOMES IN OCTOGENARIANS RECEIVING MECHANICAL THROMBECTOMY FOR LARGE VESSEL OCCLUSION ACUTE ISCHEMIC STROKE}

F Chin*, M Waqas, H Shallwani, H Rai, E Levy, A Siddiqui. Endovascular Neurosurgery, SUNY Buffalo, Buffalo, NY

\subsection{6/neurintsurg-2019-SNIS. 175}

Introduction By 2050, the number of octogenarians will triple that in 2015. Currently over a third of stroke cases occur in patients aged 80 years or older. A better understanding of stroke intervention in octogenarians is warranted considering a rapidly aging population. Here we aimed to identify risk factors predictive of favorable outcomes in octogenarian stroke patients who received mechanical thrombectomy intervention.

Materials and methods A retrospective analysis was conducted on all consecutive patients who underwent mechanical thrombectomy between January 2012 to May 2017 at the Gates Vascular Institute in Buffalo, NY. Eighty-eight octogenarian patients with large vessel occlusion were identified. These patients were divided into two groups, those with favorable outcome (mRS 0-2 at 90 days) and those with unfavorable outcome (mRS 3-6 at 90 days). Demographic, procedural, radiographic and outcome data was collected. Variables including but not limited to comorbidities, smoking status, time between symptom onset to hospital arrival, infarct core, initial NIHSS score, admission glucose, creatinine, albumin, IV tPA administration, general anesthesia, procedure type, clot location and etc. were compared between the two groups.

Results Of the 88 octogenarian thrombectomies, 29 had favorable 90-day outcome (33\%) whereas 59 had unfavorable outcome $(67 \%)$. Factors that were significantly different between those with favorable and unfavorable outcome include: presence of infarct core before intervention, NIHSS score upon admission and procedure time. Of the 29 favorable outcomes, 5 presented with infarct cores (17\%) whereas 30 of the 59 unfavorable outcome patients presented with infarct cores (51\%). The median (IQR) NIHSS upon admission for the favorable outcome interventions was $12(9-15)$ in contrast to 18 (14-21.5) for unfavorable outcome cases. Mean procedure time for favorable outcomes was $54.02 \pm 31.41$ minutes compared to $68.04 \pm$ 土. 30.97 minutes for unfavorable outcomes.

Conclusion Our study suggests infarct core, initial NIHSS score and procedure time to be potential predictors of outcome in octogenarian stroke patients receiving mechanical thrombectomy. Additional research is warranted to further evaluate the significance of these predictors.

Disclosures F. Chin: None. M. Waqas: None. H. Shallwani: None. H. Rai: None. E. Levy: 1; C; Medtronic US SWIFT
PRIME Trials. 2; C; Pulsar Vascular. Advisory Board- Stryker, NeXtGen Biologics, MEDX, Cognition Medical. 4; C; Intratech Medical Ltd., NeXtGen Biologics. 6; C; Abbott Vascular for carotid training sessions. A. Siddiqui: 1; C; Cerenovus LARGE Trial, ARISE II Trial, Medtronic SWIFT PRIME and SWIFT DIRECT Trials, MicroVention FRED Trial \& CONFIDENCE Study, MUSC POSITIVE Trial, Penumbra 3D Separator Trial, COMPASS Trial etc. 2; C; Amnis Therapeutics, Boston Scientific, Canon Medical Systems USA Inc., Cerebrotech Medical. Systems Inc., Cerenovus, Claret Medical, Corindus Inc., Endostream Medical Ltd etc. 4; C; Amnis Therapeutics, Apama Medical, BlinkTBI Inc., Buffalo Technology Partners Inc, Cardinal Health, Cerebrotech Medical Systems Inc, Claret Medical, Coggnition Medical, Endostream Medical Ltd etc.

\section{E-101 ECONOMIC IMPACT OF THE FIRST PASS EFFECT (FPE) IN THE TREATMENT OF STROKE WITH THE EMBOTRAP II DEVICE (IN ARISE-II)}

${ }^{1} \mathrm{O}$ Zaidat ${ }^{*}{ }^{2} \mathrm{~J}$ Saver, ${ }^{3} \mathrm{H}$ Mattle, ${ }^{4} \mathrm{H}$ Bozorgchami, ${ }^{5} \mathrm{~A}$ Narata, ${ }^{6} \mathrm{~A}$ Yoo, ${ }^{7} \mathrm{M}$ Ribo, ${ }^{8} \mathrm{C}$ Crivera, ${ }^{9} \mathrm{H}$ Cameron, ${ }^{10} \mathrm{~T}$ Andersson. ${ }^{1}$ Department of Neurology, St. Vincent Mercy Medical Center, Toledo, OH; ${ }^{2}$ Department of Neurology, University of California, Los Angeles, Los Angeles, $C A ;{ }^{3}$ Department of Neurology, University Hospital Bern, Bern, Switzerland; Interventional Neurology, Vascular Neurology, Oregon Health and Science University, Portland, $O R$; ${ }^{5}$ Service of Radiology and Neuroradiology, University Hospital Tours, Tours, France; ${ }^{6}$ Interventional Neuroradiology/Neuroendovascular Surgery, Texas Stroke Institute, Plano, $T X_{;}{ }^{7}$ Department of Neurology, Hospital Vall d'Hebron, Barcelona, Spain; ${ }^{8}$ Health Economics and Market Access, Cerenovus, Johnson and Johnson, Irvine, $\mathrm{CA}_{;}{ }^{9}$ Cornerstone Research Group, Burlington, ON, Canada; ${ }^{10}$ Departments of Neuroradiology and Clinical Neuroscience, Karolinska University Hospital and Karolinska Institutet, Stockholm, Sweden

\subsection{6/neurintsurg-2019-SNIS.176}

Introduction The first pass effect (FPE), a measure of thrombectomy device's ability to restore near or complete revascularization (modified Thrombolysis in Cerebral Ischemia [mTICI] $\geq 2$ c) in a single pass, may be considered as a benchmark for thrombectomy devices in the treatment of acute ischemic stroke. While the FPE has been shown to be an independent predictor of good functional outcomes (modified Rankin Scale [mRS] $\leq 2$ ) and is associated with reduced 90-day mortality, the economic impact of achieving the FPE has not been assessed.

Methodology Data were obtained from the EMBOTRAP II device, ARISE-II $(n=227)$ study. FPE was defined as complete revascularization (mTICI $\geq 2 \mathrm{c}$ ) after the first pass of the EMBOTRAP II device. Patients who did not achieve complete revascularization within the ARISE-II population were excluded from the analysis. Healthcare resources included total hospital length of stay (LOS), days in the intensive care unit (ICU), standard bed days, and procedural device use (stent retrievers and aspiration devices). Costs from the literature, in 2018 USD, were applied to healthcare resources.

Results In ARISE-II, 76\% of patients $(n=172)$ achieved complete revascularization; among these patients, 53\% achieved the FPE. Among patients that achieved complete revascularization, baseline characteristics were well-balanced between patients that did or did not achieve the FPE. A significantly higher percentage of patients that achieved the FPE had good functional outcomes vs. those that did not achieve the FPE $(80.5 \%$ vs. $61.0 \%, p=0.006)$. Healthcare resource use was lower among patients that achieved the FPE. While patients that achieved the FPE required only a single 